\title{
SOME RESULTS CONCERNING FRAMES IN BANACH SPACES
}

\author{
\|\| \\ S. K. KAUSHIK
}

\begin{abstract}
A necessary and sufficient condition for the associated sequence of functionals to a complete minimal sequence to be a Banach frame has been given. We give the definition of a weak-exact Banach frame, and observe that an exact Banach frame is weak-exact. An example of a weak-exact Banach frame which is not exact has been given. A necessary and sufficient condition for a Banach frame to be a weak-exact Banach frame has been obtained. Finally, a necessary condition for the perturbation of a retro Banach frame by a finite number of linearly independent vectors to be a retro Banach frame has been given.
\end{abstract}

\section{Introduction}

Frames for Hilbert spaces were introduced by Duffin and Schaeffer [4], while addressing some deep problems in non-harmonic Fourier series. Gröchenig [6] generalized frames for Banach spaces and called them atomic decompositions. He also introduced a more general concept of Banach spaces called Banach frames. Banach frames were further studied in 1, 2, 3, 5, 7].

In the present paper, we give a necessary and sufficient condition for the associated sequence of functionals to a complete minimal sequence to be a Banach frame. Also it has been shown that a continuous linear mapping from a Banach space $E$ onto another Banach space $F$ determines a Banach frame for $F$ (Section 3). In Section 4, w-exact Banach frames has been defined and a necessary and sufficient condition for a Banach frame to be a $w$-exact Banach frame has been given. Finally, in Section 5, we considered perturbation of a retro Banach frame by a finite number of linearly independent vectors and obtained a necessary condition for the perturbed sequence to be a retro Banach frame.

\section{Preliminaries}

Throughout this paper $E$ will denote a Banach space over the scalar field $\mathbb{K}$ ( $\mathbb{R}$ or $\mathbb{C}), E^{*}$ the conjugate space of $E, u$ the canonical isomorphism of $E$ into $\left[f_{n}\right]^{*},\left[x_{n}\right]$ the closed linear span of $\left\{x_{n}\right\}$ in the norm topology of $E,\left[\widetilde{f_{n}}\right]$ the closed linear span of $\left\{f_{n}\right\}$ in the $\sigma\left(E^{*}, E\right)$-topology, $E_{d}$ and $\left(E^{*}\right)_{d}$, respectively, the associated Banach spaces of

Received February 14, 2006; Revised March 1, 2007.

2000 Mathematics Subject Classification. Primary 42C15, Secondary 46B15.

Key words and phrases. Frames, Banach frames, retro Banach frames. 
the scalars-valued sequences indexed by $\mathbb{N}$, and $\gamma_{E}(V)$ the greatest number $r$ such that the unit ball $\{f \in V:\|f\| \leq 1\}$ of $V$ is $\sigma\left(E^{*}, E\right)$-dense in the ball $\left\{f \in E^{*}:\|f\| \leq r\right\}$ of $E^{*}$.

A sequence $\left\{x_{n}\right\}$ in $E$ is said to be complete if $\left[x_{n}\right]=E$ and a sequence $\left\{f_{n}\right\}$ in $E^{*}$ is said to be total over $E$ if $\left\{x \in E: f_{n}(x)=0, n \in \mathbb{N}\right\}=\{0\}$. A pair $\left(x_{n}, f_{n}\right)$ $\left(\left\{x_{n}\right\} \subset E,\left\{f_{n}\right\} \subset E^{*}\right)$ is called a biorthogonal system if $f_{i}\left(x_{j}\right)=\delta_{i j}$ (Kronecker's delta) for all $i, j \in \mathbb{N}$ and $E$-complete biorthogonal system if it is a biorthogonal system such that $\left[x_{n}\right]=E$. A sequence $\left\{x_{n}\right\} \subset E$ is minimal if there exists a sequence $\left\{f_{n}\right\} \subset E^{*}$ such that $\left(x_{n}, f_{n}\right)$ is a biorthogonal system. If $\left(x_{n}, f_{n}\right)$ is a $E$-complete biorthogonal system, then $\left\{x_{n}\right\}$ is a complete minimal sequence and $\left\{f_{n}\right\}$ is called the associated sequence of functional (a.s.f.) to the sequence $\left\{x_{n}\right\}$.

Definition 2.1.([6]) Let $E$ be a Banach space and $E_{d}$ be an associated Banach space of scalar-valued sequences, indexed by $\mathbb{N}$. Let $\left\{f_{n}\right\} \subset E^{*}$ and $S: E_{d} \rightarrow E$ be given. The pair $\left(\left\{f_{n}\right\}, S\right)$ is called a Banach frame for $E$ with respect to $E_{d}$ if

(i) $\left\{f_{n}(x)\right\} \in E_{d}$ for each $x \in E$,

(ii) there exist positive constants $A$ and $B$ with $0<A \leq B<\infty$ such that

$$
A\|x\|_{E} \leq\left\|\left\{f_{n}(x)\right\}\right\|_{E_{d}} \leq B\|x\|_{E}, \quad x \in E
$$

(iii) $S$ is a bounded linear operator such that

$$
S\left(\left\{f_{n}(x)\right\}\right)=x, \quad x \in E .
$$

The positive constants $A$ and $B$, respectively, are called lower and upper frame bounds of the Banach frame $\left(\left\{f_{n}\right\}, S\right)$. The operator $S: E_{d} \rightarrow E$ is called the reconstruction operator (or, the pre-frame operator). The inequality (2.1) is called the frame inequality.

The Banach frame $\left(\left\{f_{n}\right\}, S\right)$ is called tight if $A=B$ and normalized tight if $A=B=1$. If removal of one $f_{n}$ renders the collection $\left\{f_{n}\right\} \subset E^{*}$ no longer a Banach frame for $E$, then $\left(\left\{f_{n}\right\}, S\right)$ is called an exact Banach frame.

The following results which are refered in this paper are listed in the form of lemmas.

Lemma 2.1.(9]) If $E$ is a Banach space and $\left\{f_{n}\right\} \subset E^{*}$ is total over $E$, then $E$ is linearly isometric to the associated Banach space $E_{d}=\left\{\left\{f_{n}(x)\right\}: x \in E\right\}$, where the norm is given by $\left\|\left\{f_{n}(x)\right\}\right\|_{E_{d}}=\|x\|_{E}, x \in E$.

Lemma 2.2. ([8]) Let $\left(\left\{f_{n}\right\}, S\right)\left(\left\{f_{n}\right\} \subset E^{*}, S: E_{d} \rightarrow E\right)$ be a Banach frame for $E$ with respect to $E_{d}$. Then $\left(\left\{f_{n}\right\}, S\right)$ is exact if and only if $f_{n} \notin\left[\widetilde{f}_{i}\right]_{i \neq n}$, for all $n$.

Proof. Suppose first that the Banach frame $\left(\left\{f_{n}\right\}, S\right)$ is exact. Then for some $n \in \mathbb{N}$, there exists no reconstruction operator $S_{0}$ such that $\left(\left\{f_{i}\right\}_{i \neq n}, S_{0}\right)$ is a Banach frame for

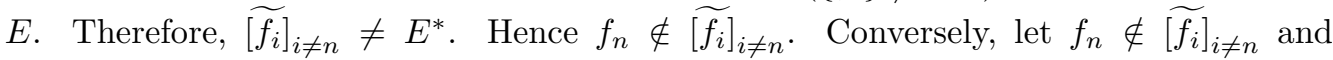
let $\left(\left\{f_{n}\right\}, S\right)$ be not exact. Then there exists a reconstruction operator $S_{1}$ defined by $S_{1}:\left(\left\{f_{i}(x)\right\}_{i \neq n}\right)=x, x \in E$ such that $\left(\left\{f_{i}\right\}_{i \neq n}, S_{1}\right)$ is a Banach frame for $E$. Therefore, by frame inequality, $\left[\widetilde{f_{i}}\right]_{i \neq n}=E^{*}$. This gives $\left.f_{n} \in \widetilde{\left[f_{i}\right.}\right]_{i \neq n}$ which is a contradiction. 

[7].

Finally, in this section, we give the definition of a retro Banach frame introduced in

Definition 2.2. Let $E$ be a Banach space and $E^{*}$ be its conjugate space. Let $\left(E^{*}\right)_{d}$ be a Banach space of scalar valued sequences associated with $E^{*}$ indexed by $\mathbb{N}$. Let $\left\{x_{n}\right\} \subset E$ and $T:\left(E^{*}\right)_{d} \rightarrow E^{*}$ be given. The pair $\left(\left\{x_{n}\right\}, T\right)$ is called a retro Banach frame $(\mathrm{RBF})$ for $E^{*}$ with respect to $\left(E^{*}\right)_{d}$ if

(i) $\left\{f\left(x_{n}\right)\right\} \in\left(E^{*}\right)_{d}$ for each $f \in E^{*}$,

(ii) there exist positive constants $A$ and $B$ with $0<A \leq B<\infty$ such that

$$
A\|f\|_{E^{*}} \leq\left\|\left\{f\left(x_{n}\right)\right\}\right\|_{\left(E^{*}\right)_{d}} \leq B\|f\|_{E^{*}}, \quad f \in E^{*}
$$

(iii) $T$ is a bounded linear operator such that $T\left(\left\{f\left(x_{n}\right)\right\}\right)=f, f \in E^{*}$.

The positive constants $A$ and $B$, respectively, are called lower and upper frame bounds of the retro Banach frame $\left(\left\{x_{n}\right\}, T\right)$. The operator $T:\left(E^{*}\right)_{d} \rightarrow E^{*}$ is called the reconstruction operator (or, the pre-frame operator). The inequality (2.2) is called the retro frame inequality.

\section{Banach frames}

We begin this section with a necessary and sufficient condition for the associated sequence of functionals to a complete minimal sequence in $E$ to be a Banach frame for $E$.

Theorem 3.1. Let $\left(x_{n}, f_{n}\right)\left(\left\{x_{n}\right\} \subset E,\left\{f_{n}\right\} \subset E^{*}\right)$ be an E-complete biorthogonal system. Then there exists an associated Banach space $E_{d}$ and a bounded linear operator $S: E_{d} \rightarrow E$ such that $\left(\left\{f_{n}\right\}, S\right)$ is a normalized tight and exact Banach frame for $E$ with respect to $E_{d}$ if and only if

$$
\lim _{n \rightarrow \infty} \alpha_{i}^{(n)}=0, i \in \mathbb{N} \quad \Longrightarrow \quad \lim _{n \rightarrow \infty} \sum_{i=1}^{m_{n}} \alpha_{i}^{(n)} x_{i}=0, i \in \mathbb{N} .
$$
$j \in \mathbb{N}$

Proof. Suppose $\lim _{n \rightarrow \infty} \alpha_{i}^{(n)}=0$ for each $i \in \mathbb{N}$ and $x=\lim _{n \rightarrow \infty} \sum_{i=1}^{m_{n}} \alpha_{i}^{(n)} x_{i}$. Then for each

$$
f_{j}(x)=\lim _{n \rightarrow \infty} \sum_{i=1}^{m_{n}} \alpha_{i}^{(n)} f_{j}\left(x_{i}\right)=\lim _{n \rightarrow \infty} \alpha_{j}^{(n)}=0 .
$$

Therefore, by frame inequality for the Banach frame $\left(\left\{f_{n}\right\}, S\right), x=0$.

Conversely, let $x \in E$ be such that $f_{j}(x)=0$ for all $j \in \mathbb{N}$. Since $\left[x_{n}\right]=E$,

$$
x=\lim _{n \rightarrow \infty} \sum_{i=1}^{p_{n}} \beta_{i}^{(n)} x_{i} \text { and so for all } j \in \mathbb{N}, \quad \lim _{n \rightarrow \infty} \beta_{j}^{(n)}=f_{j}(x)=0 .
$$


Then, by hypothesis, $x=0$. Therefore, by Lemma 2.1, there exists an associated Banach space $E_{d}=\left\{\left\{f_{n}(x): x \in E\right\}\right\}$ and a bounded linear operator $S: E_{d} \rightarrow E$ given by $S\left(\left\{f_{n}(x)\right\}\right)=x, x \in E$ such that $\left(\left\{f_{n}\right\}, S\right)$ is a normalized tight Banach frame for $E$ with respect to $E_{d}$. Further since $f_{i}\left(x_{j}\right)=\delta_{i j}$, for all $i, j \in \mathbb{N}, f_{n} \notin\left[\widetilde{f}_{i}\right]_{i \neq n}$. Indeed, if $f_{n} \in\left[\widetilde{f}_{i}\right]_{i \neq n}$, then each $f_{n}$ has the form

$$
f_{n}=\sigma\left(\left[x_{n}\right]^{*},\left[x_{n}\right]\right)-\lim _{p \rightarrow \infty} \sum_{\substack{i=1 \\ i \neq n}}^{m_{p}} \alpha_{i}^{(p)} f_{i} .
$$

Then $f_{n}\left(x_{n}\right)=0$, a contradiction. Hence, by Lemma 2.2, $\left(\left\{f_{n}\right\}, S\right)$ is an exact Banach frame for $E$ with respect to $E_{d}$.

Let $v$ be a continuous linear mapping from $E$ onto another Banach space $F$. If $E$ has a Banach frame, then the following theorem shows that we may have a Banach frame for $F$.

Theorem 3.2. Let $\left(\left\{f_{n}\right\}, S\right)\left(\left\{f_{n}\right\} \subset E^{*}, S: E_{d} \rightarrow E\right)$ be a Banach frame for $E$ with respect to $E_{d}$. Let $\left\{g_{n}\right\} \subset F^{*}$, where $F$ is any other Banach space. If there exists a continuous linear mapping $v$ from $E$ onto $F$ such that $v^{*}\left(g_{n}\right)=f_{n}, n \in \mathbb{N}$. Then there exists an associated Banach space $F_{d}$ and a bounded linear operator $U: F_{d} \rightarrow F$ such that $\left(\left\{g_{n}\right\}, U\right)$ is a normalized tight Banach frame for $F$ with respect to $F_{d}$. Moreover, if $\left(\left\{f_{n}\right\}, S\right)$ is exact, then $\left(\left\{g_{n}\right\}, U\right)$ is also exact.

Proof. For each $y \in F$ there exists an $x \in E$ such that $v(x)=y$. Let $g_{n}(y)=0$ for all $n \in \mathbb{N}$. Then

$$
f_{n}(x)=v^{*}\left(g_{n}\right)(x)=g_{n}(v(x))=g_{n}(y)=0, \text { for all } n \in \mathbb{N} .
$$

Therefore, by frame inequality for the Banach frame $\left(\left\{f_{n}\right\}, S\right), x=0$ and so $y=0$. Then by Lemma 2.1 there exists an associated Banach space $F_{d}=\left\{\left\{g_{n}(y)\right\}: y \in F\right\}$ and a bounded linear operator $U: F_{d} \rightarrow F$ given by $U\left(\left\{g_{n}(y)\right\}\right)=y, y \in F$ such that $\left(\left\{g_{n}\right\}, U\right)$ is a normalized tight Banach frame for $F$ with respect to $F_{d}$. Further since the Banach frame $\left(\left\{f_{n}\right\}, S\right)$ is exact, by Lemma 2.2. $f_{n} \notin\left[\widetilde{f}_{i}\right]_{i \neq n}$, for all $n \in \mathbb{N}$. Therefore there exists a sequence $\left\{x_{n}\right\} \subset E$ such that $f_{i}\left(x_{j}\right)=\delta_{i j}$, for all $i, j \in \mathbb{N}$. Put $y_{n}=v\left(x_{n}\right)$, $n \in \mathbb{N}$. Then $\left\{y_{n}\right\} \subset F$ is such that

$$
g_{i}\left(y_{j}\right)=g_{i}\left(v\left(x_{j}\right)\right)=v^{*}\left(g_{i}\right)\left(x_{j}\right)=f_{i}\left(x_{j}\right)=\delta_{i j}, \quad i, j \in \mathbb{N} .
$$

Thus $g_{n} \notin \widetilde{\left.g_{i}\right]_{i \neq n}}$. Hence, by Lemma 2.2, $\left(\left\{g_{n}\right\}, U\right)$ is an exact Banach frame for $F$ with respect to $F_{d}$.

Let $\left(\left\{f_{n}\right\}, S\right)\left(\left\{f_{n}\right\} \subset E^{*}, S: E_{d} \rightarrow E\right)$ be an exact Banach frame for $E$ with respect to $E_{d}$. Then, in view of Lemma 2.2 , there exists a unique sequence $\left\{x_{n}\right\} \subset E$, called the admissible sequence to the Banach frame $\left(\left\{f_{n}\right\}, S\right)$, such that for all $i, j \in \mathbb{N}, f_{i}\left(x_{j}\right)=\delta_{i j}$. 
If $\sup _{1 \leq n<\infty}\left\|\sum_{i=1}^{n} \alpha_{i} f_{i}\right\|<\infty$, then the following result shows that $\alpha_{i}$ 's are determined by $f\left(x_{i}\right)$ for each $i \in \mathbb{N}$, provided $\gamma_{\left[x_{n}\right]}\left[f_{n}\right]>0$.

Theorem 3.3. Let $\left(\left\{f_{n}\right\}, S\right)\left(\left\{f_{n}\right\} \subset E^{*}, S: E_{d} \rightarrow E\right)$ be an exact Banach frame for $E$ with respect to $E_{d}$ and with the admissible sequence $\left\{x_{n}\right\} \subset E$ such that $\gamma_{\left[x_{n}\right]}\left[f_{n}\right]>0$ and $\sup _{1 \leq n<\infty}\left\|\sum_{i=1}^{n} \alpha_{i} f_{i}\right\|<\infty$. Then there exists an $f \in E^{*}$ such that $f\left(x_{n}\right)=\alpha_{n}$, $n \in \mathbb{N}$.

Proof. Let $W$ be the natural canonical embedding of $\left[f_{n}\right]$ into $\left[u\left(x_{n}\right)\right]^{*}$. Since $\left[u\left(x_{n}\right)\right]$ is separable, the $\sigma\left(\left[u\left(x_{n}\right)\right]^{*},\left[u\left(x_{n}\right)\right]\right)$-topology is metrizable on bounded sets of $\left[u\left(x_{n}\right)\right]^{*}\left([10]\right.$, Theorem 3.1.1). Therefore there exists a subsequence $\left\{\sum_{i=1}^{n_{k}} \alpha_{i} W\left(f_{i}\right)\right\}_{k}$ of $\left\{\sum_{i=1}^{n} \alpha_{i} W\left(f_{i}\right)\right\}_{n}$ which is $\sigma\left(\left[u\left(x_{n}\right)\right]^{*},\left[u\left(x_{n}\right)\right]\right)$-convergent to some $\psi \in\left[u\left(x_{n}\right)\right]^{*}$. Since $\gamma_{\left[x_{n}\right]}\left[f_{n}\right]>0, W$ is an isomorphism of $\left[f_{n}\right]$ onto $\left[u\left(x_{n}\right)\right]^{*}$. Therefore there exists an $f \in\left[f_{n}\right]$ such that $\psi=W(f)$.

Hence

$$
\begin{aligned}
f\left(x_{n}\right) & =(W(f))\left(u\left(x_{n}\right)\right) \\
& =\psi\left(u\left(x_{n}\right)\right) \\
& =\sigma\left(\left[u\left(x_{n}\right)\right]^{*},\left[u\left(x_{n}\right)\right]\right)-\lim _{k \rightarrow \infty} \sum_{i=1}^{n_{k}} \alpha_{i} W\left(f_{i}\right)\left(u\left(x_{n}\right)\right) \\
& =\alpha_{n}, \quad n \in \mathbb{N} .
\end{aligned}
$$

\section{Weak-exact Banach frames}

Definition 4.1. A Banach frame $\left(\left\{f_{n}\right\}, S\right)\left(\left\{f_{n}\right\} \subset E^{*}, S: E_{d} \rightarrow E\right)$ for $E$ with respect to $E_{d}$ is called weak exact (in short, w-exact) if there exists a sequence $\left\{\phi_{n}\right\} \subset$ $E^{* *}$, called an admissible sequence to $\left(\left\{f_{n}\right\}, S\right)$, such that $\phi_{i}\left(f_{j}\right)=\delta_{i j}$, for all $i, j \in \mathbb{N}$.

An admissible sequence to a $w$-exact Banach frame $\left(\left\{f_{n}\right\}, S\right)$ need not be unique as in case of exact Banach frames.

Example 4.1. Let $E=l^{1}$ and let $\left\{f_{n}\right\}$ be the sequence of unit vectors in $E^{*}$. Then by Lemma 2.1 there exists an associated Banach space $E_{d}=\left\{\left\{f_{n}(x)\right\}: x \in E\right\}$ and a reconstruction operator $S: E_{d} \rightarrow E$ given by $S\left(\left\{f_{n}\right\}(x)\right)=x, x \in E$ such that $\left(\left\{f_{n}\right\}, S\right)$ is a Banach frame for $E$ with respect to $E_{d}$. Define $\left\{g_{n}\right\} \subset E^{* *}$ by

$$
g_{n}(f)=\xi_{n} \quad\left(f=\left\{\xi_{n}\right\} \in E^{*}, n \in \mathbb{N}\right) .
$$

Then $\left\{g_{n}\right\}$ is an admissible sequence to the $w$-exact Banach frame $\left(\left\{f_{n}\right\}, S\right)$. Let $e \in E^{*}$ be such that $e \notin\left[f_{n}\right]=c_{0}$. Let $g_{0} \in E^{* *}$ be such that $g_{0}(e) \neq 0$ and $g_{0}\left(\left[f_{n}\right]\right)=\{0\}$. Define $\left\{h_{n}\right\} \subset E^{* *}$ by $h_{1}=g_{1}-g_{0}, h_{n}=g_{n}, n=2,3, \ldots$ Then $h_{i}\left(f_{j}\right)=\delta_{i j}$ for all $i, j \in \mathbb{N}$. So $\left\{h_{n}\right\}$ is another admissible sequence to $\left(\left\{f_{n}\right\}, S\right)$.

We now give a necessary and sufficient condition for the uniqueness of the admissible sequence to a $w$-exact Banach frame. 
Theorem 4.1. An admissible sequence to a w-exact Banach frame $\left(\left\{f_{n}\right\}, S\right)$ is unique if and only if $\left(\left\{f_{n}\right\}, S\right)$ is a retro Banach frame for $E^{* *}$ with respect to $E_{d}$.

Proof. Let $\left\{\phi_{n}\right\} \subset E^{* *}$ be the unique admissible sequence to $\left(\left\{f_{n}\right\}, S\right)$. Suppose $\left(\left\{f_{n}\right\}, S\right)$ is not a retro Banach frame for $E^{* *}$. Then by Theorem 3.1 in $[7],\left[f_{n}\right] \neq E^{*}$. Define $\left\{\psi_{n}\right\} \subset E^{* *}$ by $\psi_{1}=\phi_{1}-\phi_{0}$ and $\psi_{n}=\phi_{n}, n=2,3, \ldots$ Then $\psi_{i}\left(f_{j}\right)=\delta_{i j}$, for all $i, j \in \mathbb{N}$. This is a contradiction.

Conversely, let $\left\{g_{n}\right\}$ and $\left\{h_{n}\right\}$ in $E^{* *}$ be two admissible sequences to the $w$-exact Banach frame $\left(\left\{f_{n}\right\}, S\right)$. Then $\left(g_{i}-h_{i}\right)\left(f_{j}\right)=\delta_{i, j}$, for all $i, j \in \mathbb{N}$. Since $\left(\left\{f_{n}\right\}, S\right)$ is a retro Banach frame for $E^{* *},\left[f_{n}\right]=E^{*}$. Hence $g_{i}=h_{i}$ for all $i \in \mathbb{N}$.

In view of Lemma 2.2 one may observe that an exact Banach frame for $E$ is a $w$-exact Banach frame for $E$. The converse however need not be true as shown by the following example.

Example 4.2. Let $E=c_{0}$. Define $\left\{f_{n}\right\} \subset E^{*}$ by

$$
\left\{\begin{array}{l}
f_{1}=(1,0,0, \ldots) \\
f_{n}=((-1)^{n+1}, 0,0, \ldots, \quad \underbrace{}_{\substack{\downarrow \\
n^{\text {th }}}} \underset{\text { place }}{1}, 0, \ldots), \quad(n=2,3, \ldots)
\end{array}\right.
$$

and $\left\{\phi_{n}\right\} \subset E^{* *}$ by

$$
\left\{\begin{array}{l}
\phi_{1}=(1,1,-1,1,-1,1, \ldots) \\
\phi_{n}=\left(0,0, \ldots, \quad \underset{\downarrow}{n^{\text {th }}} \underset{\text { place }}{ }, 0, \ldots\right), \quad(n=2,3, \ldots)
\end{array}\right.
$$

Then by Lemma 2.1 there exist an associated Banach space $E_{d}=\left\{\left\{f_{n}(x)\right\}: x \in E\right\}$ and a reconstruction operator $S: E_{d} \rightarrow E$ given by $S\left(\left\{f_{n}(x)\right\}\right)=x, x \in E$ such that $\left(\left\{f_{n}\right\}, S\right)$ is a Banach frame for $E$ with respect to $E_{d}$. Also since $\phi_{i}\left(f_{j}\right)=\delta_{i j}$ for all $i, j \in \mathbb{N}$, $\left(\left\{f_{n}\right\}, S\right)$ is a $w$-exact Banach frame. Further since $\left[f_{n}\right]=E^{*}$, by Theorem 3.1 in []], $\left(\left\{f_{n}\right\}, S\right)$ is a retro Banach frame for $E^{* *}$. Therefore, by Theorem $4.1,\left\{\phi_{n}\right\}$ is the unique admissible sequence to the $w$-exact Banach frame $\left(\left\{f_{n}\right\}, S\right)$. But $\phi_{1} \notin \pi(E)$, where $\pi$ is the canonical isomorphism of $E$ into $E^{* *}$. So there exists no sequence $\left\{x_{n}\right\} \subset E$ such that $f_{i}\left(x_{j}\right)=\delta_{i j}$, for all $i, j \in \mathbb{N}$. Hence, by Lemma $2.2,\left(\left\{f_{n}\right\}, S\right)$ is not exact.

We now give a necessary and sufficient condition for a Banach frame to be $w$-exact.

Theorem 4.2. Let $\left(\left\{f_{n}\right\}, S\right)\left(\left\{f_{n}\right\} \subset E^{*}, S: E_{d} \rightarrow E\right)$ be a Banach frame for $E$ with respect to $E_{d}$. Then $\left(\left\{f_{n}\right\}, S\right)$ is $w$-exact if and only if

$$
\lim _{n \rightarrow \infty} \sum_{i=1}^{m_{n}} \alpha_{i}^{(n)} f_{i}=0 \quad \Rightarrow \quad \lim _{n \rightarrow \infty} \alpha_{i}^{(n)}=0, \text { for each } i
$$


Proof. Suppose $\left\{\phi_{n}\right\} \subset E^{* *}$ be an admissible sequence to the $w$-exact Banach frame $\left(\left\{f_{n}\right\}, S\right)$. Let $\lim _{n \rightarrow \infty} \sum_{i=1}^{m_{n}} \alpha_{i}^{(n)} f_{i}=0$. Then for each $j \in \mathbb{N}$,

$$
\lim _{n \rightarrow \infty} \alpha_{j}^{(n)}=\lim _{n \rightarrow \infty} \sum_{i=1}^{m_{n}} \alpha_{i}^{(n)} \phi_{j}\left(f_{i}\right)=0 .
$$

Conversely, let $Q=\left\{\sum_{i=1}^{n} \alpha_{i} f_{i}: f_{i} \in E^{*}, \alpha_{i} \in \mathbb{K} ; i \in \mathbb{N}\right\}$. Let $\left\{g_{n}\right\} \subset E^{* *}$ be such that for each $j \in \mathbb{N}, g_{j}\left(\sum_{i=1}^{\bar{n}} \alpha_{i} f_{i}\right)=\alpha_{j}$. If $f \in \bar{Q}$, the closure of $Q$, then $f=$ $\lim _{n \rightarrow \infty} \sum_{i=1}^{m_{n}} \alpha_{i}^{(n)} f_{i}$. So, by hypothesis, $g_{j}(f)=\lim _{n \rightarrow \infty} \alpha_{j}^{(n)}$ for each $j \in \mathbb{N}$. Thus, for each $j, g_{j}$ is a continuous linear functional on $\bar{Q}$. Also $g_{j}\left(f_{i}\right)=\delta_{i j}$, for all $i, j \in \mathbb{N}$. Hence $\left(\left\{f_{n}\right\}, S\right)$ is $w$-exact.

\section{Perturbation of Banach frames}

Let $\left(\left\{f_{n}\right\}, S\right)\left(\left\{f_{n}\right\} \subset E^{*}, S: E_{d} \rightarrow E\right)$ be a Banach frame for $E$ with respect to $E_{d}$ and let $f_{0}$ be a nonzero functional in $E^{*}$. If $\left(\left\{f_{n}\right\}, S\right)$ is an exact Banach frame for $E$, then for a non-zero functional $f_{0} \in E^{*}$, the following example shows that there may exists a reconstruction operator $S_{0}$ such that $\left(\left\{f_{n}+f_{0}\right\}, S_{0}\right)$ is a Banach frame for $E$ which is not exact.

Example 5.1. Let $E=c_{0}$. Define $\left\{h_{n}\right\} \in E^{*}$ by

$$
h_{n}(x)=\xi_{n}, \quad x=\left\{\xi_{j}\right\} \in E .
$$

Then by Lemma 2.1 there exists an associated Banach space $E_{d}=\left\{\left\{h_{n}(x)\right\}: x \in E\right\}$ and a bounded linear operator $S: E_{d} \rightarrow E$ such that $\left(\left\{h_{n}\right\}, S\right)$ is a normalized tight exact Banach frame for $E$ with respect to $E_{d}$. Now observe that the sequence $\left\{h_{n}+h_{1}\right\} \subset E^{*}$ is total over $E$. Therefore, again by Lemma 2.1 there exists an associated Banach space $E_{d_{1}}=\left\{\left\{\left(h_{n}+h_{1}\right)(x)\right\}: x \in E\right\}$ and a reconstruction operator $S_{1}: E_{d_{1}} \rightarrow E$ such that $\left(\left\{h_{n}+h_{1}\right\}, S_{1}\right)$ is a Banach frame for $E$ with respect to $E_{d_{1}}$. Further by Lemma 2.2 $\left(\left\{h_{h}+h_{1}\right\}, S_{1}\right)$ is not exact since $\left[\widetilde{h_{n}+h_{1}}\right]_{n \neq 1}=E^{*}$.

It is natural to ask under what condition the Banach frame $\left(\left\{h_{n}+h_{1}\right\}, S_{1}\right)$ is exact. The following theorem answers this query.

Theorem 5.1. Let $\left(\left\{f_{n}\right\}, S\right)\left(\left\{f_{n}\right\} \subset E^{*}, S: E_{d} \rightarrow E\right)$ be an exact Banach frame for $E$ with admissible sequence $\left\{x_{n}\right\} \subset E$ such that $\left[x_{n}\right]=E$. Let $f_{0}$ be a non-zero functional in $E^{*}$. If there exists an associated Banach space $E_{d_{0}}$ and a reconstruction operator $S_{0}: E_{d_{0}} \rightarrow E$ such that $\left(\left\{f_{n}+f_{0}\right\}, S_{0}\right)$ is a Banach frame for $E$, then the Banach frame $\left(\left\{f_{n}+f_{0}\right\}, S_{0}\right)$ is non-exact.

Proof. Since $\left\{x_{n}\right\} \subset E$ is an admissible sequence to the Banach frame $\left(\left\{f_{n}\right\}, S\right)$, $f_{i}\left(x_{j}\right)=\delta_{i j}$, for all $i, j \in \mathbb{N}$. Suppose $\left(\left\{f_{n}+f_{0}\right\}, S_{0}\right)$ is exact. Then, by Lemma 2.2 , there exists a sequence $\left\{y_{n}\right\} \subset E$ such that $\left(f_{i}+f_{0}\right)\left(y_{j}\right)=\delta_{i j}$, for all $i, j \in \mathbb{N}$. Since 
$f_{0} \neq 0$, there exists a $p \in \mathbb{N}$ such that $f_{0}\left(x_{p}\right) \neq 0$. Let $m \geq p$ be a fixed but arbitrary integer and $\alpha_{1}, \alpha_{2}, \ldots, \alpha_{m}$ be any scalars. Then

$$
\begin{aligned}
\left|\sum_{i=1}^{m} \alpha_{i}\left(f_{i}+f_{0}\right)\left(x_{p}\right)\right| & =\left|\alpha_{p}+\sum_{i=1}^{m} \alpha_{i} f_{0}\left(x_{p}\right)\right| \\
& \geq\left|\sum_{i=1}^{m} \alpha_{i}\right|\left|f_{0}\left(x_{p}\right)\right|-\left|\sum_{i=1}^{m} \alpha_{i}\left(f_{i}+f_{0}\right)\left(y_{p}\right)\right| .
\end{aligned}
$$

This gives

$$
\left|\sum_{i=1}^{m} \alpha_{i}\right| \leq\left(\left\|x_{p}\right\|+\left\|y_{p}\right\|\right)\left(f_{0}\left(x_{p}\right)\right)^{-1}\left\|\sum_{i=1}^{m} \alpha_{i}\left(f_{i}+f_{0}\right)\right\| .
$$

Therefore, by Helly's theorem (11], p.109), there exists an element $x \in E$ such that $\left(f_{i}+f_{0}\right)(x)=1$, for all $i \in \mathbb{N}$. Put $y=x /\left(1-f_{0}(x)\right)$. Then $y \in E$ is such that $f_{i}(y)=1$, for all $i \in \mathbb{N}$. Let $f \in E^{*}$ be such that $f(y) \neq 0$. Put $f_{0}=((-1) / f(y)) f$. Then $0 \neq f_{0} \in E^{*}$ is such that $\left(f_{i}+f_{0}\right)(y)=0$, for all $i \in \mathbb{N}$. This is a contradiction.

In the following theorem, we consider perturbation of a retro Banach frame (RBF) by a finite number of linearly independent elements and obtain a necessary condition for the perturbed sequence to be a RBF.

Theorem 5.2. Let $\left(\left\{x_{n}\right\}, U\right)\left(\left\{x_{n}\right\} \subset E, U:\left(E^{*}\right)_{d} \rightarrow E^{*}\right)$ be a $R B F$ for $E^{*}$ with respect to $\left(E^{*}\right)_{d}$. Let $\left\{z_{k}\right\}_{k=1}^{m}$ be a linearly independent set of vectors in $E$ and let for each $k(1 \leq k \leq m)$ there exists an $f_{k} \in E^{*}$ such that $f_{k}\left(x_{n}\right)=c_{k}^{(n)}$, for all $n \in \mathbb{N}$. If $\left(\left\{x_{n}+\sum_{k=1}^{m} c_{k}^{(n)} z_{k}\right\}, V\right)$ is a RBF for $E^{*}$ with respect to $\left(E^{*}\right)_{d}$, where $V:\left(E^{*}\right)_{d} \rightarrow E^{*}$ is a reconstruction operator, then -1 is not an eigen value of the matrix

$$
\left(\begin{array}{cccc}
f_{1}\left(z_{1}\right) & f_{2}\left(z_{1}\right) & \ldots & f_{m}\left(z_{1}\right) \\
f_{1}\left(z_{2}\right) & f_{2}\left(z_{2}\right) & \ldots & f_{m}\left(z_{2}\right) \\
\vdots & \vdots & \vdots & \vdots \\
f_{1}\left(z_{m}\right) & f_{2}\left(z_{m}\right) & \ldots & f_{m}\left(z_{m}\right)
\end{array}\right)
$$

Proof. It is enough to prove the result for the case $m=2$. Suppose -1 is an eigenvalue of the matrix

$$
\left(\begin{array}{ll}
f_{1}\left(z_{1}\right) & f_{2}\left(z_{1}\right) \\
f_{1}\left(z_{2}\right) & f_{2}\left(z_{2}\right)
\end{array}\right)
$$

Then

$$
\left|\begin{array}{cc}
f_{1}\left(z_{1}\right)+1 & f_{2}\left(z_{1}\right) \\
f_{1}\left(z_{2}\right) & f_{2}\left(z_{2}\right)+1
\end{array}\right|=0
$$

So there exists scalars $\alpha, \beta$ not both zero, such that

$$
\begin{aligned}
& \alpha f_{1}\left(z_{1}\right)+\beta f_{2}\left(z_{1}\right)=-\alpha \\
& \alpha f_{1}\left(z_{2}\right)+\beta f_{2}\left(z_{2}\right)=-\beta .
\end{aligned}
$$


Put $g=-\alpha f_{1}-\beta f_{2}$. Then, $g$ is a non-zero functional in $E^{*}$ such that

$$
g\left(x_{n}\right)=-\alpha c_{1}^{(n)}-\beta c_{2}^{(n)}, \text { for all } n \in \mathbb{N},
$$

where $c_{i}^{(n)}=f_{i}\left(x_{n}\right), i=1,2$.

Now

$$
g\left(z_{k}\right)=-\alpha f_{1}\left(z_{k}\right)-\beta f_{2}\left(z_{k}\right), \quad k=1,2 .
$$

Therefore $g\left(z_{1}\right)=\alpha$ and $g\left(z_{2}\right)=\beta$. Thus

$$
g\left(x_{n}+c_{1}^{(n)} z_{1}+c_{2}^{(n)} z_{2}\right)=0, \quad \text { for all } n \in \mathbb{N} .
$$

Since $\left(\left\{x_{n}+\sum_{k=1}^{2} c_{k}^{(n)} z_{k}\right\}, V\right)$ is a RBF for $E^{*}$, it follows from the retro frame inequality that $g=0$. This is a contradiction.

As an application to the above theorem, we give the following example.

Example 5.2. Let $E=c_{0}$ and let $\left\{x_{n}\right\} \subset E$ be the sequence of unit vectors. Then, by Lemma 2.1, there exists an associated Banach space $\left(E^{*}\right)_{d}=\left\{\left\{f\left(x_{n}\right)\right\}: f \in E^{*}\right\}$ and a reconstruction operator $U:\left(E^{*}\right)_{d} \rightarrow E^{*}$ such that $\left(\left\{x_{n}\right\}, U\right)$ is a RBF for $E^{*}$ with respect to $\left(E^{*}\right)_{d}$.

Let $z_{1}, z_{2} \in E$ be given by

$$
z_{1}=(1,0,0, \ldots), \quad z_{2}=(0,1,0, \ldots)
$$

and let $f_{1}, f_{2} \in E^{*}$ be given by

$$
f_{1}=(-1,0,0, \ldots), \quad f_{2}=(0,-1,0, \ldots) .
$$

Then -1 is an eigen value of the matrix

$$
\left(\begin{array}{ll}
f_{1}\left(z_{1}\right) & f_{2}\left(z_{1}\right) \\
f_{1}\left(z_{2}\right) & f_{2}\left(z_{2}\right)
\end{array}\right)
$$

Therefore, by Theorem 5.2, there exists no reconstruction operator $V:\left(E^{*}\right)_{d} \rightarrow E^{*}$ such that $\left(\left\{x_{n}+\sum_{k=1}^{2} c_{k}^{(n)} z_{k}\right\}, V\right)$ is a $\mathrm{RBF}$ for $E^{*}$ with respect to $\left(E^{*}\right)_{d}$, where $c_{k}^{(n)}=$ $f_{k}\left(x_{n}\right), k=1,2$.

\section{Acknowledgements}

The author thanks the reviewer for making suggestions for the improvement of the paper. 


\section{References}

[1] P. G. Casazza, D. Han and D. R. Larson, Frames for Banach spaces, Contemp. Math. 247(1999), 149-182.

[2] P. G. Casazza and O. Christensen, Perturbation of operators and applications to frame theory, J. Fourier Anal. Appl. 3(1997), 543-557.

[3] O. Christensen and C. Heil, Perturbation of Banach frames and Atomic decompositions, Math. Nachr. 185(1997), 33-47.

[4] R. J. Duffin and A. C. Schaeffer, A class of non-harmonic Fourier series, Trans. Amer. Math. Soc. 72(1952), 341-366.

[5] S. J. Favier and R. A. Zalik, On the stability of Frames and Riesz bases, Appl. Comp. Harm. Anal. 2(1995), 160-173.

[6] K. Gröchenig, Describing functions : Atomic decompositions versus frames, Monatsh. Math. 112(1991), 1-41.

[7] P. K. Jain, S. K. Kaushik and L. K. Vashisht, Banach frames for conjugate Banach spaces, Zeit. Anal. Anwendungen. 23(2004), 713-720.

[8] P. K. Jain, S. K. Kaushik and L. K. Vashisht, On Banach frames, Indian J. Pure \& Appl. Math. 37(2006), 265-272.

[9] I. Singer, Bases in Banach spaces-II, Springer - Verlag, New York - Heidelberg, 1981.

[10] A. Wilansky, Topolgy for Analysis. Robert E. Krieger Publishing Co. Inc., 1983.

[11] K. Yoshida, Functional Analysis, Springer - Verlag, Berlin - Heidelberg - New York, 1966.

Department of Mathematics, Kirorimal College (University of Delhi), Delhi, India.

E-mail: shikk2003@yahoo.co.in 